\title{
Surgical care and COVID-19 pandemic
}

\author{
Komal Gupta ${ }^{1}$, Supreet Kaur ${ }^{1}$, Aditya Baksi ${ }^{2}$, Niladri Banerjee ${ }^{2}$, Kamal Kataria ${ }^{1 *}$
}

\begin{abstract}
${ }^{1}$ Department of Surgical Disciplines, All India Institute of Medical Sciences, New Delhi, India
${ }^{2}$ Department of Surgery, All India Institute of Medical Sciences, Jodhpur, Rajasthan, India
\end{abstract}

\author{
Received: 27 March 2021 \\ Accepted: 10 May 2021 \\ *Correspondence: \\ Dr. Kamal Kataria, \\ E-mail: drkamalkataria@gmail.com
}

Copyright: () the author(s), publisher and licensee Medip Academy. This is an open-access article distributed under the terms of the Creative Commons Attribution Non-Commercial License, which permits unrestricted non-commercial use, distribution, and reproduction in any medium, provided the original work is properly cited.

\begin{abstract}
The COVID-19 pandemic has added a huge strain on health care resources globally. For the developing world and low-to-middle income countries (LMIC), the burden has been even greater. Patients with COVID-19 require proper isolation and treatment. However, patients with other medical and surgical conditions should not be denied emergency treatment. While elective surgeries may be deferred, emergency surgeries should not wait. Therefore, hospitals should have a well-defined plan to allocate their resources judiciously, at the same time ensuring a safe ecosystem for health care workers and patients. Triage of patients attending the emergency department can ensure better delivery of health care in a resource-constrained set up. Based on our experience, we have formulated an algorithm for management of surgical emergencies during the COVID-19 pandemic. Guidelines from various surgical societies were referred to, and modified according to the needs and constraints of a LMIC.
\end{abstract}

Keywords: COVID-19, Emergency, Healthcare worker, LMIC, Pandemic, Surgery

\section{INTRODUCTION}

In December 2019, a cluster of cases of pneumonia were reported in Wuhan, China, which soon became an epidemic across the whole country. In February 2020 the world health organization (WHO) designated it as Coronavirus disease 2019 (COVID-19) and the responsible pathogen as severe acute respiratory syndrome Coronavirus 2 (SARS-CoV-2), and in March 2020 , it was declared as a pandemic. ${ }^{1}$ The disease is transmitted from person to person via aerosols generated during coughing, sneezing and via fomites. ${ }^{2}$ SARS-CoV2 has been so far isolated from fecal cultures, gastrointestinal epithelium and blood. ${ }^{3,4}$ All body tissues and fluids should be considered as potential sources of the virus..$^{5}$ The gold standard for diagnosis of the disease is made with a positive reverse transcriptase polymerase chain reaction (RT-PCR) test for SARS-CoV-2 performed on upper respiratory tract specimens. ${ }^{6}$ High rates of false negative result, especially during the early asymptomatic phase and the recovery phase of the disease, indicate that the patient can still transmit the disease. ${ }^{7}$ Therefore, COVID-19 suspects are to be dealt with the same level of infection control measures as diagnosed positive cases.

In response to COVID-19, countrywide curfews and restrictions on movement were implemented to slow down the spread of the disease. Hospitals across the globe had put a halt on routine care to divert hospital staff to cater to the needs of critically ill COVID-19 patients. As per the guidelines from most surgical societies across the globe, all elective surgical procedures were postponed until a control on the pandemic was achieved. ${ }^{8}$

The All-India institute of medical sciences, New Delhi, is a tertiary care hospital catering to both primary and referred cases from other hospitals in North India. The busy emergency department (ED) has three separate divisions for medical emergencies, surgical emergencies and trauma care. The ED area has a triage counter and designated red, yellow and green areas. Though the 
burden and pattern of cases presenting to the ED had considerably reduced and changed during the nationwide curfew, as the restrictions eased out, there was a surge of cases. ${ }^{9}$ It cannot be overemphasized that the principles of patient management remained the same as pre-pandemic times. The main goals were to provide necessary care to all patients in a timely manner, while ensuring safety of healthcare professionals and fellow patients from contracting SARS-CoV-2 infection and to ensure optimal utilization of resources.

Based on our experience, we have formulated an algorithm to manage patients presenting with surgical emergencies in an optimal manner from door to discharge. It focuses on specific considerations over and above the usual patient management protocol to prevent transmission of SARS-CoV-2, keeping in mind the needs and constraints of low-middle income countries (LMIC).

\section{EMERGENCY DEPARTMENT SETUP}

\section{Red area}

It is to be subdivided into hot, warm, and cold contamination zones. ${ }^{10}$

\section{Hot zone}

The area in immediate vicinity of patient trolleys should be marked with a red tape to indicate the contaminated area. This area should include the patient trolley, monitor units and Mayo stand with essential supplies for airway, breathing and circulation (ABC) management. Personnel in this area should donn appropriate personal protective equipment (PPE). Number of personnel entering this zone should be minimized to prevent the overuse of PPE. Number of personnel is subject to change on a case-tocase basis. It can be decided according to prior emergency medical services (EMS) information or at triage counter. All non-essential supplies should be removed from the hot zone. The team leader should not enter the hot zone unless necessary. X-ray and ultrasound equipment kept in hot zone should be covered with disposable drapes. Plexiglass shield should be used for Xray machine if available.

\section{Warm zone}

It is at a distance of three feet from the hot zone and is marked by yellow tape. Personnel without appropriate PPE should avoid entering this area. A transfer table should be placed in the warm zone to supply the equipment required in the hot zone.

\section{Cold zone}

This is an area beyond the warm zone, outside the yellow tape. All the supplies are to be stocked in the cold zone. Personnel not directly involved in patient care should stay in this zone. The team leader should direct activities in the hot zone from the cold zone.

\section{MANAGEMENT OF INCOMING PATIENTS}

\section{Triage}

Patients are received at the ED triage counter. Assessment is done as per usual triage protocol into red, yellow, and green categories. If a patient is stable, a COVID-19 screening questionnaire is filled at the time of triage, which classifies patients based on presence or absence of symptoms and/or possible contact with COVID-19 infected person. A patient is labeled as 'COVID suspect' or 'non COVID suspect' according to COVID-19 screening criteria. Patients with compromised primary survey/ABC are triaged to red area without filling COVID-19 screening questionnaire and are considered as 'COVID suspect' until proven otherwise. Essential lifesaving care should not be delayed for determining COVID-19 status. Non-COVID suspect patients should receive care as per standard protocol by healthcare workers wearing appropriate PPE. To prevent unnecessary attendance at ED, early involvement of senior staff members and definitive decision-making should be performed early in the point of contact of the patients.

\section{Strategy for diagnosed COVID-19 patients and COVID- 19 suspects}

\section{Primary survey}

Airway maintenance with cervical spine protection: Donning of appropriate PPE is essential for all healthcare personnel involved in COVID suspect areas. If endotracheal intubation is needed, rapid sequence intubation (RSI) is preferred. ${ }^{11}$ Duration of bag and mask ventilation should be limited; if required, a rebreathing mask should be used for oxygenation. Intubation at the first attempt should be the goal. Intubation should be done by the most experienced individual present in the hot zone. Video laryngoscopy, if available should be preferred. Disposable equipment should be used. Nondisposable equipment should be cleaned with freshly prepared $0.1 \%$ sodium hypochlorite solution, and later appropriately sterilized. ${ }^{12}$ The face of the patient should be covered with transparent plastic drapes during intubation. A closed suction system, if available, should be used for aspiration and suctioning post endotracheal intubation. ${ }^{13}$ The endotracheal tube cuff should be fully inflated before connecting it to the mechanical ventilator. For cricothyroidotomy, the same principles as endotracheal intubation should be followed and transparent plastic drapes should be used above the surgical site.

Breathing: Wide bore cannula, used for needle thoracostomy, in patients with tension pneumothorax should be connected to the underwater seal before 
insertion into the chest cavity to minimize aerosolization into the patient's vicinity as the air under high pressure is released on needle decompression. Similarly, induction of cough occurs after pleural breach during tube thoracostomy leading to aerosolization, hence it has to be performed with extra caution. ${ }^{14}$ If the patient is on mechanical ventilation, then appropriate sedation or paralysis should be given to prevent sudden coughing. Removal of intercostal drain (ICD) also needs precaution especially when the patient is on mechanical ventilator. Ventilation should be on hold and removal should be done only after putting an occlusive dressing on three sides of the entry site. Complete sealing of ICD incision site should be done to prevent pneumothorax formation.

Circulation with bleeding control: The patient should be resuscitated with fluids. Blood products should be used judiciously.

Disability assessment: Airway should be secured if GCS is less than 8 , in the manner described above.

Exposure and environmental control: During exposure of the patient, all clothes and personal belongings are to be handled by personnel who have donned appropriate PPE, as fomites are potential sources of transmission of SARSCoV-2.

\section{Investigations}

Chest X-ray or ultrasonography of chest should be considered in all patients to detect features of pneumonitis including those patients who require immediate surgery. Appropriate infection control protocol should be formulated to avoid hospital related transmission of COVID-19 in the radiology suites.

\section{MANAGEMENT}

\section{Preoperative planning}

Decision regarding operative management should be made by two senior surgeons, who should agree to the need of operative intervention. Non-operative management (NOM), whenever possible, should be considered. The least invasive and least aerosol generating procedure (AGP) should be chosen. The anticipated length of hospital stay must be considered while making management decisions, e.g., total duration of close observation should be considered for NOM. Day care surgery should be considered if possible. Enhanced recovery after surgery (ERAS) protocols should be implemented whenever possible to ensure early discharge from hospital, thus, limiting the total exposure of health care professionals and use of hospital resources especially PPE. ${ }^{16}$ Preoperative risk stratification tools should be used for prognostication. Patients should be assessed early by a senior anesthetist.
Ideally, all patients who are planned for surgery, intervention or admission to ward/ ICU, irrespective of COVID-19 suspicion index, should be considered for COVID-19 RTPCR testing before proceeding. However, this may not always be feasible due to resource and time constraints.

Patients with diagnosed SARS-CoV-2 infection should be operated in a designated COVID Operation Room (COR). Patients requiring emergency surgery (who cannot wait for RTPCR results) and patients with compromised primary survey and non-responder status (where COVID screening with questionnaire cannot be performed), should be considered as COVID suspects and operated in COR. During resuscitation of these patients before shifting to COR, oral/nasopharyngeal swab collection may be attempted. However, emergency surgery should not be delayed waiting for results of RTPCR. Patients without suspicion of COVID (no evidence based on symptoms/USG/Chest-X ray) with negative RTPCR, as well as COVID suspects (evidence based on symptoms/USG/Chest X-ray) with a negative RT-PCR, should undergo surgery with standard operative room protocol. Anesthetists should use N-95/FFP3 mask and face shield during intubation. ${ }^{15}$ If delay in surgery does not compromise the quality of care, then surgery in COVID suspects should be performed when result of RTPCR is available, to prevent wastage of resources, and preferably at daytime, when staff and resources are optimal.

\section{Urgent/emergent surgeries}

Urgent surgery should be considered for life-threatening emergencies like intestinal obstruction, perforation, intestinal ischemia, massive gastrointestinal bleed not controlled by endoscopic measures, and sepsis with surgically amenable septic foci. Conservative management may be attempted for adhesive small bowel obstruction. Cases of intussusception can be managed by pneumatic and/or hydrostatic enemas with appropriate precautions. Threshold for stoma formation (as opposed to anastomosis) should be low to decrease chances of postoperative complications. Decision regarding management of postoperative complications associated with anastomosis leak in sick patients leading to prolonged hospital stay and need for repeat surgery should be made after appropriate discussion with senior surgeons and investigations like CT scan.

\section{Cancer and elective surgeries}

Management of cancers requiring surgical intervention needs to be decided on a case-to-case basis. While delay in treatment can adversely affect survival of patients with cancer, decisions have to be taken keeping in mind that hospitals have mobilized their resources to cater for the intensive care and sub-intensive care needs of COVID-19 patients. Cancer patients are at high risk of severe COVID-19 and related mortality due to their 
immunocompromised status. Hospital visits and hospitalization being potential sources for cross-infection of SARS-CoV-2 amongst patients, there is an ethical dilemma in cancer management. A multi-disciplinary team should make the decision about appropriate treatment protocol for each patient. Patients should be made aware of the factors determining their treatment, the obstacles in acquiring certain aspects of the treatment and an informed decision should be made based on it. General principals of oncological management, however, remain the same. Non-deferrable cancer patients will require surgery on priority basis. Surgeries for breast reconstruction and prophylactic surgeries should be deferred preferably. Management of pheochromocytoma and paraganglioma requires availability of ICU and an experienced anesthesiology team, which may not always be available in a pandemic situation. Delaying surgery in such patients can precipitate life-threatening emergencies; therefore, appropriate medical management is required till surgery is performed.

\section{Consent}

Patients and families should be informed about the goals of care, possible COVID-related complications, and need for strict isolation, if the patient is diagnosed as SARSCoV-2 positive. Relatives should be communicated by phone, if not possible in person. Documentation of the consent should be completed in the ED before shifting the patient to the operating room.

\section{COVID OR set up}

The dedicated COVID OR should have negative-pressure air-flow with high frequency air exchange facility of more than 25 per hour. ${ }^{17}$ Dedicated donning/ doffing area and shower area should be connected to the OR. All essential supplies should be ready before starting the procedure. Number of surgical items should be limited to only what is essential. Entry and exit from the COR should be restricted. Doors of the OR should be closed at all times. If the OR has two doors, one should be reserved for access to materials brought to the OR and another for entry of patient and donned surgical staff. All surgical items should be placed on a table, to be picked up by the staff inside the room. Personnel handling used surgical instruments (e.g., OR technician) should donn appropriate PPE (face shield, double gloves and N95/FFP3 mask). All surfaces should be cleaned after each operation with $0.1 \%$ sodium hypochlorite, or as per local guidelines. All surgical instruments should be covered for transport and returned for immediate sterilization following case completion. Disposable alternatives for instruments and supplies should be used if possible.

\section{Shifting to $O R$}

Patients should be shifted to COT via dedicated 'COVID pathway' including dedicated 'COVID lifts'. All preoperative documentation should be completed prior to shifting to OR. Patients should wear face mask if not intubated. Care teams should coordinate over phone prior to transport. Peer to peer handover must occur upon arrival of the patient to the COR. Staff transporting COVID-19 positive or suspected patients to the OR must wear PPE, which is to be doffed upon leaving the OR. Surgeon and scrub nurse should enter the OR only after donning of appropriate PPE including gown, double gloves, face shield, shoe covers, head cover and N95/ FFP3 mask.

\section{Anesthesia considerations}

Donning of full PPE is essential for all healthcare personnel involved. Regional anesthesia should be preferred whenever feasible. Duration of bag-mask ventilation should be limited. Appropriate filters should be attached to the mask, which should be well-fitted to the patient's face, to avoid leaks and aerosolization. Change of gloves should be done immediately after endotracheal intubation to limit possible spread of the virus.

\section{Surgical approach}

Efforts should be made to reduce operative time. Surgeries should preferably be performed by senior surgeons. Enhanced recovery after surgery (ERAS) protocols should be followed whenever feasible. Use of appropriate thromboprophylaxis and antibiotic prophylaxis and minimally invasive surgical approaches should be encouraged. Prophylactic use of nasogastric tubes and surgical drains should be avoided. ${ }^{18}$ Use of electrocautery, laser or ultrasonic scalpel should be limited to reduce aerosol generation. ${ }^{19}$ Exposure to surgical plumes should be avoided as much as possible. Surgical plume contains cellular debris, blood cells and certain viruses. Though currently there is no definitive evidence of presence of SARS-CoV-2 in surgical plumes, appropriate precautions should be maintained. Smoke evacuators or filtration devices and a closed suction system should be used. Surgical team changes should be made in prolonged surgeries, as working for long duration in full PPE is tiresome. In case of exposure of scrubbed personnel decontamination should be done according to CDC guidelines.

\section{Laparoscopy}

No evidence of increased risk of SARS-CoV-2 transmission with laparoscopic surgery (compared with open) has been found. ${ }^{20}$ Laparoscopy significantly decreases the rate of postoperative complications and leads to early discharge, thus decreasing exposure to health care personnel. However, if laparoscopic approach is anticipated to considerably increase operative time (e.g., pancreato-duodenectomy), open surgery may be preferred. ${ }^{21}$ Port site incisions should not be longer than the diameter of the port, in order to prevent gas leakage. 
Intra-abdominal pressure should be kept as low as possible to reduce gas leak. ${ }^{22}$ Hand assisted surgery should be avoided. Smoke evacuator attached to a portable HEPA filtration device should be used. Pneumoperitoneum should be completely evacuated via a portable HEPA filtration system before removing ports. ${ }^{23}$ Patients should recover from anesthesia in the COR itself as coughing and vomiting during recovery period increases the chances of aerosolization. Post-operative documentation should be done outside the OR. Peer-topeer sign out should be done and the patient should return to the isolation ward or COVID ICU without holdup in the postoperative recovery area. Multidisciplinary care of these patients with involvement of physicians and surgeons should be done. COVID-19 related medical complications should be managed as per standard protocols.

\section{Discharge and follow up}

At the time of discharge, the patient should be provided with a follow-up plan. Patient and attendants should be trained regarding relevant wound care, stoma care or tracheostomy care adequately before discharge. Duration of all medications should be mentioned clearly in the discharge summary. Use of telemedicine should be done for routine or emergency follow up and patients should be given clear instructions about the use of telemedicine.

\section{CONCLUSION}

The COVID-19 pandemic has added a strain of magnanimous proportions for most of the developing world and low-middle income countries (LMIC), which were already functioning on a hand-to-mouth basis. This is where the role of triage comes in. Although patients with COVID-19 require immediate attention, patients with other medical and surgical conditions should not be denied treatment. Hospitals should have a well-defined plan ready to allocate their resources judiciously, and constantly update their patient management protocols to maintain a safe ecosystem for health care workers and patients.

Funding: No funding sources Conflict of interest: None declared

Ethical approval: Not required

\section{REFERENCES}

1. Cucinotta D, Vanelli M. WHO Declares COVID-19 a Pandemic. Acta Biomed. 2020;91(1):157-60.

2. Van Doremalen N, Bushmaker T, Morris DH. Aerosol and Surface Stability of SARS-CoV-2 as Compared with SARS-CoV-1. N Engl J Med. 2020;382(16):1564-7.

3. Cheung KS, Hung IFN, Chan PPY. Gastrointestinal Manifestations of SARS-CoV-2 Infection and Virus Load in Fecal Samples from a Hong Kong Cohort:
Systematic Review and Metaanalysis. Gastroenterology. 2020;159(1):81-95.

4. Chen W, Lan Y, Yuan X. Detectable 2019-nCoV viral RNA in blood is a strong indicator for the further clinical severity. Emerg Microbes Infect. 2020;9(1):469-73.

5. Kutti-Sridharan G, Vegunta R, Vegunta R, Mohan BP, Rokkam VRP. SARS-CoV2 in Different Body Fluids, Risks of Transmission, and Preventing COVID-19: A Comprehensive Evidence-Based Review. Int J Prev Med. 2020;11:97.

6. Goudouris ES. Laboratory diagnosis of COVID-19. J Pediatr (Rio J). 2021;97(1):7-12.

7. Long DR, Gombar S, Hogan CA. Occurrence and Timing of Subsequent SARS-CoV-2 RT-PCR Positivity Among Initially Negative Patients. Clin Infect Dis. 2020; ciaa722.

8. Di Martino M, García Septiem J, Maqueda González R. Elective Surgery During the SARS-CoV-2 Pandemic (COVID-19): A Morbimortality Analysis and Recommendations on Patient Prioritisation and Security Measures. Cir Esp. 2020;S0009739X(20)30166-4.

9. Park C, Sugand K, Nathwani D, Bhattacharya R, Sarraf KM. Impact of the COVID-19 pandemic on orthopedic trauma workload in a London level 1 trauma center: the "golden month". Acta Orthop. 2020;1-6.

10. Livingston DH, Bonne S, Morello C, Fox A. Optimizing the trauma resuscitation bay during the covid-19 pandemic. Trauma Surg Acute Care Open. 2020;5(1):e000488.

11. Meng L, Qiu H, Wan L. Intubation and Ventilation amid the COVID-19 Outbreak: Wuhan's Experience. Anesthesiology. 2020;132(6):1317-32.

12. Kampf G, Todt D, Pfaender S, Steinmann E. Persistence of coronaviruses on inanimate surfaces and their inactivation with biocidal agents. J Hosp Infect. 2020;104(3):246-51.

13. Broderick D, Kyzas P, Sanders K, Sawyerr A, Katre C, Vassiliou L. Surgical tracheostomies in Covid-19 patients: important considerations and the "5Ts" of safety. Br J Oral Maxillofac Surg. 2020;58(5):585-9.

14. Pieracci FM, Burlew CC, Spain D. Tube thoracostomy during the COVID-19 pandemic: guidance and recommendations from the AAST Acute Care Surgery and Critical Care Committees. Trauma Surg Acute Care Open. 2020;5(1):e000498.

15. Dau NQ, Peled H, Lau H, Lyou J, Skinner C. Why N95 Should Be the Standard for All COVID-19 Inpatient Care [published online ahead of print, 2020 Jun 29]. Ann Intern Med. 2020;M20-2623.

16. Sica GS, Campanelli M, Bellato V, Monteleone G. Gastrointestinal cancer surgery and enhanced recovery after surgery (ERAS) during COVID-19 outbreak. Langenbecks Arch Surg. 2020;405(3):3578.

17. Wong J, Goh QY, Tan Z. Preparing for a COVID-19 pandemic: a review of operating room outbreak 
response measures in a large tertiary hospital in Singapore. Can J Anaesth. 2020;67(6):732-45.

18. Ni X, Jia D, Guo Y, Sun X, Suo J. The efficacy and safety of enhanced recovery after surgery (ERAS) program in laparoscopic digestive system surgery: A meta-analysis of randomized controlled trials. Int $\mathbf{J}$ Surg. 2019;69:108-15.

19. Karuppal R, Surendran S, Patinharayil G, Muhammed Fazil VV, Marthya A. It is time for a more cautious approach to surgical diathermy, especially in COVID-19 outbreak: A schematic review. J Orthop. 2020;20:297-300.

20. Vigneswaran Y, Prachand VN, Posner MC, Matthews JB, Hussain M. What Is the Appropriate Use of Laparoscopy over Open Procedures in the Current COVID-19 Climate? J Gastrointest Surg. 2020;24(7):1686-91.

21. Sheng S, Zhao T, Wang X. Comparison of robotassisted surgery, laparoscopic-assisted surgery, and open surgery for the treatment of colorectal cancer: A network meta-analysis. Medicine (Baltimore). 2018;97(34):e11817.

22. Pini Prato A, Conforti A, Almstrom M. Management of COVID-19-Positive Pediatric Patients Undergoing Minimally Invasive Surgical Procedures: Systematic Review and Recommendations of the Board of European Society of Pediatric Endoscopic Surgeons. Front Pediatr. 2020;8:259.

23. Lin LW, Hung TY. Swivel-HEPA-ETT (SHE) bougie and HEPA-ETT (HE) methods for safe intubation while managing patients with COVID19. Emerg Med J. 2020;37(5):256-7.

Cite this article as: Gupta K, Kaur S, Baksi A, Banerjee N, Kataria K. Surgical care and COVID-19 pandemic. Int Surg J 2021;8:1959-64. 